\title{
Benefits of Neoadjuvant Hypofractionated Radiotherapy in the Treatment of Locally Advanced Adenocarcinomas
}

\author{
Mourad Abid $^{1 *}$, Mohamed Amine Mansouri ${ }^{1}$, Abderraouf Bataiche ${ }^{1}$, Yousri Ballah ${ }^{1}$, Mourad Brahimi ${ }^{1}$, Zakia Kordjani $^{1}$ and Abdelhalim Hammani ${ }^{2}$ \\ ${ }^{1}$ Department of Oncologic Surgery, Anti-Cancer Center, Batna, Algeria \\ ${ }^{2}$ Department of Oncologic Surgery, Centre Pierre et Marie Curie, Algiers, Algeria \\ *Corresponding author: Mourad Abid, Department of Oncologic Surgery, Anti-Cancer Center, Batna, Algeria, Tel: 00213770421615; E- \\ mail: abidmourad@hotmail.com
}

Received date: June 29, 2017; Accepted date: July 03, 2017; Published date: July 07, 2017

Copyright: (c) 2017 Abid M. This is an open access article distributed under the terms of the Creative Commons Attribution License, which permits unrestricted use, distribution, and reproduction in any medium, provided the original author and source are credited.

\begin{abstract}
Background: The current treatment of locally advanced lower and middle rectum's adenocarcinoma is well codified. It is based on neoadjuvant radiotherapy (NRT) followed by surgical excision. However, two broad radiotherapy approaches are being debated: short course of hypo-fractionated radiation therapy "SRT" (5 GY $\times 5$ days) and long course concomitant radio-chemotherapy "CRT" using higher radiation dose (45-50 GY over 5 weeks) combined with chemotherapy. The first approach allows taking care of about 4 times more patients compared to the second in a same period. SRT was introduced at a large scale at the Pierre ET Marie Curie center (Algiers) in May 2010 by a new multidisciplinary board (Rectum board), aiming to reduce long-lasting schedules.
\end{abstract}

Purpose: The aim of the current study is to evaluate the SCR introduction effect on the access rate to NRT and also to define its impact on treatment efficacy by assessing the primary tumor response (downsizing and downstaging) and the complete resection margin $\mathrm{RO}$ rates.

Patients and methods: In this study, the feasibility of short-course radiotherapy was evaluated. A specimen from 297 patients was studied over 10 years. We took May 2010 as a reference point to divide this specimen into two groups, group 1 in care before May $2010(n=130)$ and group 2 treated after May $2010(n=167)$. Three therapeutics protocols were used: long-course chemoradiation, short-course radiotherapy and long-course radiotherapy.

Results: In the group 1, only $33 \%(43 / 130)$ of patients received NRT (SRT=2.5\%, CRT=60.5\%, LCR $=37 \%$ ). In the second group (after May 2010), 65\% (113/167) of patients were able to access an NRT (SRT $=73 \%$, CRT $=21 \%$, $\mathrm{LCR}=6 \%)(\mathrm{p}<0.001)$. Complete response (ypTON0) was obtained in 13 patients $(30 \%)$ of Group 1 and 2 patients $(2 \%)$ of group $2(p<0.001)$.

Conclusions: The large scaled introduction of short course radiotherapy and the creation of the rectum board have allowed doubling the number of patients which benefited from neoadjuvant radiotherapy. Although, the complete responses rate had significantly dropped.

Keywords: Preoperative radiotherapy; Hypofractionated radiotherapy; Concomitant radio-chemotherapy; Rectal cancer

\section{Introduction}

Colorectal cancer is the third most common cancer in the world, with nearly 1.4 million new cases diagnosed in 2012, over 746000 new cases per year for men (10\% of all cancers) and 614000 new cases per year for women $(9.2 \%$ of all cancers). There is a large geographic difference in the global distribution of colorectal cancer. About 54 per cent of colorectal cancer cases occurred in more developed countries. The highest incidence of colorectal cancer was in Oceania and Europe and the lowest incidence in Africa and Asia [1]. In Algeria, about 6000 new cases had been diagnosed every year [2,3]. However, this incidence is increasing rapidly over the lasts decades, it had almost quadrupled passing from 6 new cases per 100000 people per year in 1993 to 23.4 new cases per 100000 for men in 2013 according to Algiers cancer registry.
The current treatment of locally advanced lower and middle rectum's adenocarcinoma is based on neoadjuvant radiotherapy (NRT) combined or not to chemotherapy, followed by a carcinological surgery excision, total mesorectal excision. The indications of such treatment are: sub serosa invasion $(\mathrm{T} 3, \mathrm{~T} 4)$ and/or lymph node metastasis $\left(\mathrm{N}^{+}\right)$ [4-6].

There are two radiation regimens typically used in clinical practice are still being debated such as hypo fractionated radiotherapy so-called short course radiation "SRT" (5 fractions of 5 Gy delivered over 1 week) and long course concomitant radio-chemothera py "CRT" using higher radiation dose (45-50 GY over 5 weeks) combined with chemotherapy. This debate is mainly centered on how to get the most optimal beneficial effect with the lowest toxicity. In developed countries, CRT followed by surgery within 4-8 weeks is the gold standard because of its excellent results demonstrated in local control of the disease leading to total eradication of all Tumor cells in some cases $[7,8]$ (complete response) thus prompting some authors to suggest exclusive treatment by RT without surgery [9]. 
Citation: Abid M, Mansouri MA, Bataiche A, Ballah Y, Brahimi M, et al. (2017) Benefits of Neoadjuvant Hypofractionated Radiotherapy in the Treatment of Locally Advanced Adenocarcinomas. J Gen Pract (Los Angel) 5: 315. doi:10.4172/2329-9126.1000315

Page 2 of 5

The surprising increase in colorectal cancer incidence in Algeria has reduced the capacities of treatment by chemoradiation due to lack of radiotherapy facilities. A committee (rectum committee) created in May 2010 suggested treating patients with hypofractionated radiotherapy which would theoretically increase the patient management capacity by fourfold The aim of this study is to evaluate the efficacy of this approach.

\section{Patients and Methods}

This retrospective study comprised 297 eligible patients with middle and lower rectal cancer, enrolled between May 2004 and January 2010 in Debussy clinic of Algiers before and after the creation of the rectum committee on May 2010 in order to evaluate the efficacy of their treatment. Individual patient data were collected: Age, gender, medical history, American Society of Anesthesiology score (ASA), TNM staging (clinic, endoscopic, MRI, scanner), neoadjuvant and adjuvant treatment and distance between the lower pole of the tumor and the anal margin. All included patients had locally advanced rectal cancer (T3, T4 or node positive disease) part of them had received Neoadjuvant radiotherapy. A summary of all the data concerning the two groups are shown in the (Table 1).

\begin{tabular}{|l|l|l|l|}
\hline \multicolumn{2}{|l|}{ Characteristics } & Group 1 (n=130) & Group 2 (n=167) \\
\hline Middle Age & 55 years & 55 years \\
\hline Gender M/W\% & $73 / 130(56 \%)$ & $97 / 167(58 \%)$ \\
\hline \multirow{2}{*}{ location } & Middle $(5-10 \mathrm{~cm})$ & $88(68 \%)$ & $112(67 \%)$ \\
\hline ASA 1+2 & $42(32 \%)$ & $52(33 \%)$ \\
\hline Liver metastases & $128(98 \%)$ & $165(99 \%)$ \\
\hline Adjuvant Chemotherapy & $18(13.8 \%)$ & $13(7.8 \%)$ \\
\hline Short-course radiotherapy & $41(31.5 \%)$ & $55(33 \%)$ \\
\hline Chemo radiation & $1(2.5 \%)$ & $81(73 \%)$ \\
\hline Long-course radiotherapy & $26(60.5 \%)$ & $23(21 \%)$ \\
\hline
\end{tabular}

Table 1: Characteristics of patients before (Group 1) and after (Group 2) May 2010.

In this study 130 patients were treated before May 2010 (group 1) and 167 after (group 2), the main objectives were to evaluate the access rate to neoadjuvant radiotherapy, the tumor response (downsizing and down staging) and the completion of R0 resection. The Secondary endpoints were tumor resectability, severe postoperative morbidity (grade III and IV according to Clavian and Dindo classification), postoperative mortality and local recurrence (Figure 1).

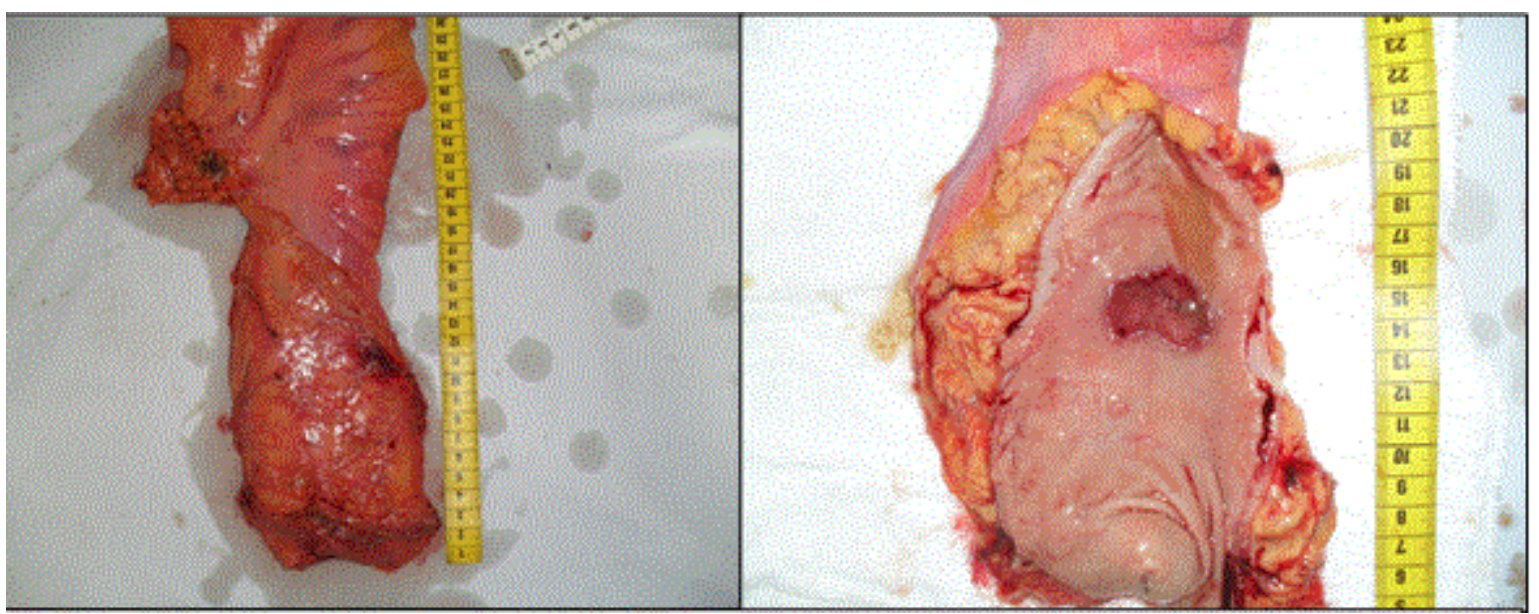

Figure 1: Down staging chemo radiation+surgery (TME) after 6 weeks of a tumor initially classified cT3N+. After histological study, it is classified ypT2N0. 
Citation: Abid M, Mansouri MA, Bataiche A, Ballah Y, Brahimi M, et al. (2017) Benefits of Neoadjuvant Hypofractionated Radiotherapy in the Treatment of Locally Advanced Adenocarcinomas. J Gen Pract (Los Angel) 5: 315. doi:10.4172/2329-9126.1000315

Page 3 of 5

Three radiation regimens had been used: chemo radiation (CRT) 45-50 Gy in 5 weeks combined with adjuvant chemotherapy using $5 \mathrm{FU}$, long-course radiotherapy (LCR) without chemotherapy and short-course radiotherapy 5 Gy in 5 days (SRT) (total of 25 Gy) (Figure 2).

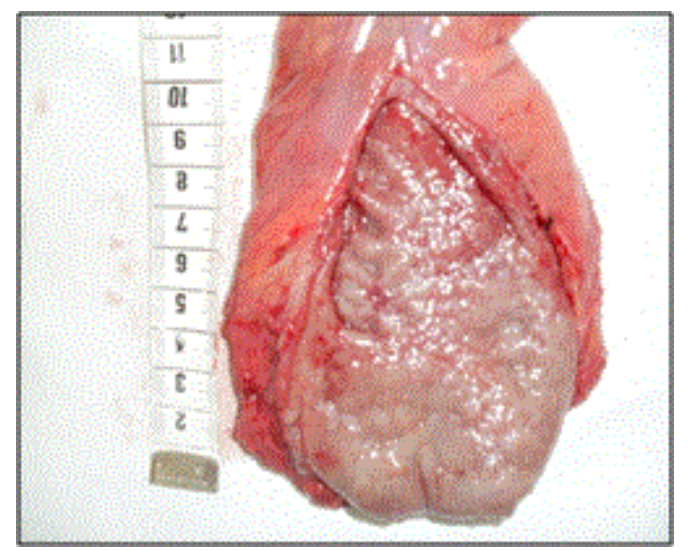

Figure 2: Complete sterilization after short course radiotherapy. Tumor initially classified cT3N.

\section{Results}

Before May 2010, only 33\% (43/130) of patients have been treated by neoadjuvant radiotherapy ( $\mathrm{SCR}=2.5 \%, \mathrm{CR}=60.5 \%, \mathrm{LCR}=37 \%$ ). In the second phase (after May 2010), 65\% (113/167) of patients have been able to access neoadjuvant radiotherapy ( $\mathrm{SCR}=73 \%$; $\mathrm{CR}=21 \%$; $\mathrm{LCR}=6 \%$ ). The use of short-course radiotherapy has doubled the number of patients treated. Surgical excisions were performed on 113 patients in group $1(87 \%)$ and on 138 patients in group 2 (83\%). 53 abdominoperineal resection (47\%) has been achieved in group 1 and $52(38 \%)$ in group $2(\mathrm{p}=0.1)$, the rest of surgical operations were low anterior resection with or without restoring the intestinal continuity (Figure 1).

The R0 (complete resection) rate in group 1 and 2 was $90 \%$ $(102 / 113)$ and $85.5 \%(118 / 138)$, respectively. This difference is not significant $(\mathrm{p}=0.3)$. The neoadjuvant treatment response was assessed after histological study by comparing the ypTNM classification with the inceptive cTNM classification. 13 patients $(30 \%)$ in group 1 had a pathological complete response (ypT0N0) versus 2 patients $(2 \%)$ in group $2(\mathrm{p}<0.001)$. Furthermore, 21 patients in group $1(49 \%)$ had a down staging ypT1-2N0 and 39 patients $(35 \%)$ in group $2,(\mathrm{p}=0.1)$.

Postoperative complication was reported in 45 of 130 patients (35\%) in group 1 versus 36 in the second group (21.5\%) ( $\mathrm{p}=0.01)$; reminding that complications counted are those which required a surgical or instrumental action (hemorrhagic or septic complication) or with an organ failure treated in intensive care unit. The difference in mortality was not significant, $7 \%$ in group $1(n=9)$ versus $5 \%$ in group $2(n=9)$ $(\mathrm{p}=0.5)$. In the other hands, the difference in local recurrence rate was significant with $17 \%$ in group 1 and $9 \%$ in group $2(\mathrm{p}=0.05)$ (Table 2$)$.

\begin{tabular}{|l|l|l|l|}
\hline & Group 1 (n=130) & Group 2 (n=167) & $\mathbf{P}$ \\
\hline Neoadjuvant radiotherapy & $43(33 \%)$ & $111(67 \%)$ & $<0.001$ \\
\hline Anterior Resection & $113(87 \%)$ & $138(83 \%)$ & 0.5 \\
\hline Abdominoperineal resection & $53(47 \%)$ & $52(38 \%)$ & 0.1 \\
\hline Complete resection margins R0 & $102(90 \%)$ & $118(85.5 \%)$ & 0.3 \\
\hline Morbidity III-IV & $45(35 \%)$ & $36(21 \%)$ & 0.01 \\
\hline Mortality & $9(7 \%)$ & $9(5 \%)$ & 0.5 \\
\hline Local recurrence & $19(17 \%)$ & $12(9 \%)$ & 0.05 \\
\hline Complete response T0 N0 & $13 / 43(30 \%)$ & $2 / 111(2 \%)$ & $<0.001$ \\
\hline Downstaging T1-2 / N0 & $21(49 \%)$ & $39(35 \%)$ & 0.1 \\
\hline
\end{tabular}

Table 2: Postoperative complication results.

\section{Discussion}

Neoadjuvant radiotherapy in the management of rectal cancer is used if the tumor center is located within $10 \mathrm{~cm}$ from anal margin and infiltration has exceeded muscularis propria and/or reached lymph nodes. Despite the clarity of these indications, not all of patients were able to access neoadjuvant radiotherapy due to lack of radiotherapy accelerators in Algeria before 2010 [10]. By creating the rectum committee and adopting the short-course radiotherapy we have been able to recruit more patients. The Colorectal Cancer Collaborative Group and Wong et al. conducted literature-based meta-analyses that focused on preoperative radiation, and they found beneficial effects on local control compared to surgery alone $[11,12]$. Then, meta-analyses by De Caluwé et al. and Ceelen et al. demonstrated that preoperative CRT improves local control in resectable stage II and III rectal cancer compared to preoperative RT alone [13,14]. Nevertheless a metaanalyses by Chen et al. based on eight randomized controlled trials involving 6894 patients found that short course radiotherapy substantially reduced the risk of local recurrence compared with surgery alone or selective postoperative radiation \pm chemotherapy, also this effect is also comparable when compared with long course chemo 
radiation, which is in contrast with the meta-analysis findings by De Caluwé et al. and Ceelen et al. [15].

Although SRT has been shown to marginally improve survival over surgery alone in this meta-analysis, it must be emphasized that the Swedish trial was the only included trial that reported a significant improvement in survival, and there was evidence of heterogeneity across the included studies [16]. Therefore, this result should be interpreted with caution. Contrary to the belief that reduction in local recurrence after radiation increases the likelihood of overall survival, no significant effect in terms of overall survival was found from SRT approach compared with selective postoperative radiation \pm chemotherapy [17]. The result was similar with prior meta-analyses [11-14]. This finding seems to be reasonable, considering the following facts. First, the reduction in mortality from rectal cancer may be largely counterbalanced by the increased risk of death from other causes. Second, the number of patients may not be large enough to reliably detect small but significant differences in long-term survival. Furthermore, Chen et al. did not find a statistically significant difference in overall survival between the SRT group and the long course chemo radiation group. Also some trials have demonstrated their similarity in terms of recurrence-free survival [18].

In the matter of our primary endpoint of judgment, we were able to double the number of patients receiving neoadjuvant radiotherapy from $33 \%$ to $65 \%$ in a shorter time span of 2 years. This result was comforting as the logistics platform remained unchanged with the same number of accelerators as before 2010. In addition to that as the smaller number of fractions makes short course radiation (SRT) less expensive and more convenient than long course radiation, this schedule is preferred in countries with limited financial resources.

The R0 resection rate was comparable to the published literature, approaching 90\% which suggest that the 6-8 weeks delay we have set for surgery is also feasible with short-course radiotherapy [19].

The benefit of radiotherapy is not limited in long-term local control of the disease, as was shown in an update of the German Trial after 11 years of follow-up [20]. One of the main consequences is its capability to lead to tumor regression on different levels: the size of primary tumor (downsizing) and the depth of wall and nodes infiltration (downstaging). In Sanghera et al. trial, $42 \%$ of complete response after chemoradiation was observed [21]. It has decreased in our study from $30 \%$ (before May 2010) to 2\% (after May 2010) after adoption of the short scheme which is not astounding given the lower doses and time compared with long scheme. However, more than the third of patients has downstaging which is satisfying and approaching the results of different series published. However, In An interim analysis of the Stockholm III trial comparing 3 preoperative radiotherapy schemes showed a pathologic complete response (pCR) in $12.5 \%$ of patients in the short course radiation with delayed-surgery group whereas the rate of pCR after short course radiotherapy with immediate surgery was only $0.8 \%$. As shown in other nonrandomized studies $[7,8]$, the rate of pCR after short course radiation and delayed surgery is not largely different from that which is observed after conventionally fractionated chemoradiation. Therefore, short course radiation with a delayed interval to surgery can be a reasonable option for elderly patients with comorbidities, who are often unfit for chemotherapy and can be used when tumour shrinkage is necessary prior to resection [15].

The postoperative morbidity was defined as complication occurring between 0 and 30 days after the surgical procedure. It included septic complications (anastomotic leak, wound infection, evisceration and deep abscess), hemorrhagic complications and postoperative ileus. Overall morbidity rate was $21 \%$ in group 2 versus $35 \%$ in group 1 with statistical significance $(p=0.01)$; this result could be distorted since we did not collect all complications (grade I and II of Clavien-dindo) and we also didn't differentiate side effects of radiotherapy from those of surgical procedure. The different published studies did not show any difference in morbidity after the two protocols [21]. Nevertheless, Chen et al. demonstrated that in the SRT group, 8 of 318 patients (2.5\%) developed a grade 3 or 4 acute treatment related toxicity, while this grade of toxicity was observed in 75 out of 320 patients (23.4\%) in the CRT group. This difference was statistically significant (RR 0.11; 95\% CI, 0.05 to $0.22 ; \mathrm{P}<0.00001)$. There was no statistically significant heterogeneity among the studies $(\mathrm{I} 2=44 \%, \mathrm{P}=0.18)$ which shows the superiority of SRT in terms of reducing post-radiotherapy morbidity.

As for local recurrence, no statistically significant difference in local recurrence was observed in the short course irradiation group when compared with the chemoradiation group in Chen et al. meta-analyses. In our study we did notice a significant difference between the two groups, $17 \%$ in group 1 and $9 \%$ in group 2 . This encouraging and comforting result contains however a statistical bias. In fact, the followup time were not similar, the first group was followed for 5 years while the term of the second group was only 3 years. We consider these results satisfying since we have been able to give population in need access to this vital treatment. However, we have to recognize some methodological criticisms. Indeed, the retrospective characters of our study decrease its argument value. The shorter follow-up time of group 2 has deprived the results of an important element, the local recurrence that we could not compare to literature.

\section{Conclusion}

Outcomes continue to improve in colorectal cancer as affected patients are discovered earlier in the disease process, largely attributable to increased screening efforts. Improved surgical technique and the incorporation of pre-operative radiotherapy all appear to confer additional benefits for a large portion of patients. On its side the cancer management in Algeria has confronted a lot of challenges by lack of organization and logistical means. The introduction of hypofractionated radiotherapy had doubled the number of patients treated with neo-adjutant radiotherapy. We also reported a decrease in postoperative morbidity and a better local control of the disease. Further studies are needed to better evaluate this approach using better control of the scientific parameters.

\section{Implications and Limitations}

What are the implications of this study? The "hypofractionated" radiotherapy allows to multiply by three the capacity to treat patients with advanced localized rectum cancers in a specialized center in Algeria. There are limitations to this study; First, the heterogeneous groups of patients and second, the retrospective design. Therefore, results should be interpreted with caution, in particular when looking at the "local" recurrences number and the rate of histological response after radiotherapy.

\section{References}

1. Ferlay J, Soerjomataram I, Ervik M, Dikshit R, Eser S, et al. (2014) GLOBOCAN 2012 Estimated Cancer incidence and mortality prevalence worldwide. 
Citation: Abid M, Mansouri MA, Bataiche A, Ballah Y, Brahimi M, et al. (2017) Benefits of Neoadjuvant Hypofractionated Radiotherapy in the Treatment of Locally Advanced Adenocarcinomas. J Gen Pract (Los Angel) 5: 315. doi:10.4172/2329-9126.1000315

Page 5 of 5

2. Hammouda D (2013) Epidemiology of colorectal cancers, national program for the detection of colorectal cancer.

3. Zimmermann F, Molls M (2003) Indications for neoadjuvant therapy in rectal carcinoma. Chirurg 74: 887-896.

4. Huffington AL (2016) Cancer of the rectum and colon in progression from year to year in Algeria.

5. Benson AB, Venook AP, Bekaii-Saab T, Chan E, Chen YJ, et al. (2015) Rectal cancer, version 2.2015. J. Natl Compr Canc Netw 13: 719-728.

6. National thesaurus of digestive cancers (TNCD) (2013) Cancer du rectum.

7. Radu C, Berglund A, Påhlman L, Glimelius B (2008) Short course preoperative radiotherapy with delayed surgery in rectal cancer: A retrospective study. Radiother Oncol 87: 343-349.

8. Hatfield, P Hingorani M, Radhakrishna G, Cooper R, Melcher A, et al. (2009) Short-course radiotherapy, with elective delay prior to surgery, in patients with unresectable rectal cancer who have poor performance status or significant comorbidity. Radiother Oncol 92: 210-214.

9. Habr-Gama A, Perez RO, Nadalin W, Sabbaga J, Ribeiro U Jr, et al. (2004) Operative versus nonoperative treatment for stage 0 distal rectal cancer following chemoradiation therapy: Long-term results. Ann Surg 240: 711-717.

10. http://www.sante.gov.dz/plan\%20cancer/plan_national_cancer.pdf

11. Colorectal Cancer Collaborative Group (2001) Adjuvant radiotherapy for rectal cancer:a systematic overview of 8507 patients from 22 randomised trials. Lancet 358: 1291-1304.

12. Wong RK, Tandan V, De Silva S, Figueredo A (2007) Pre-operative radiotherapy and curative surgery for the management of localized rectal carcinoma. Cochrane Database of Syst Rev 18: CD002102.

13. De Caluwé L, Van Nieuwenhove Y, Ceelen WP (2013) Preoperative chemoradiation versus radiation alone for stage II and III resectable rectal cancer. Cochrane Database Syst Rev 28: CD006041.
14. Ceelen W, Fierens K, Van Nieuwenhove Y, Pattyn P (2009) Preoperative chemoradiation versus radiation alone for stage II and III resectable rectal cancer. Int J Cancer 124: 2966-2972.

15. Chen C, Sun P, Rong J, Weng HW, Dai QS, et al. (2015) Short course radiation in the treatment of localized rectal cancer: A systematic review and meta-analysis. Sci Rep 5: 10953.

16. Swedish Rectal CT (1997) Improved survival with preoperative radiotherapy in resectable rectal cancer. N Engl J Med 336: 980-987.

17. Kapiteijn E, Marijnen CA, Nagtegaal ID, Putter H, Steup WH, et al. (2001) Preoperative radiotherapy combined with total mesorectal excision for resectable rectal cancer. N Engl J Med 345: 638-646.

18. Bujko K, Nowacki MP, Nasierowska-Guttmejer A, Michalski W, Bebenek $\mathrm{M}$, et al. (2006) Long-term results of a randomized trial comparing preoperative short-course radiotherapy with preoperative conventionally fractionated chemoradiation for rectal cancer. Br J Surg 93: 1215-1223.

19. Guckenberger M, Saur G, Wehner D, Sweeney RA, Thalheimer A, et al. (2012) Comparison of preoperative short-course radiotherapy and longcourse radio chemotherapy for locally advanced rectal cancer. Strahlenther Onkol 188: 551-557.

20. Sauer R, Liersch T, Merkel S, Fietkau R, Hohenberger W, et al. (2012) Preoperative versus postoperative chemoradiotherapy for locally advanced rectal cancer: Results of the German CAO/ARO/AIO-94 randomized phase III trial after a median follow-up of 11 years. J Clin Oncol 30: 1926-1933.

21. Sanghera P, Wong DW, McConkey CC, Geh JI, Hartley A (2008) Chemo radiotherapy for rectal cancer: An updated analysis of factors affecting pathological response. Clin Oncol 20: 176-183. 